\title{
THE ASSOCIATION BETWEEN OCCLUSAL TRAITS AND ORAL HEALTH RELATED QUALITY OF LIFE AMONG ADULTS IN PRIMARY CARE SETTINGS IN SAUDI ARABIA
}

\author{
Shoroog Hassan Agou*
}

\begin{abstract}
Most Oral Health Related Quality of Life (OHRQoL) orthodontic research focused on examining the relationship between malocclusion/orthodontic treatment need and OHRQoL amongst children, adolescents, and occasionally young adults. Very few studies evaluated such a relationship in adults 18 years and above, despite the increased demands for orthodontic treatment among this age group. This cross-sectional epidemiological study aims to examine the relationship between five occlusal traits and perceived quality of life in adults while considering age and gender differences. Adults above 18-years-old were invited to participate during their routine visit to primary care dental centers in Jeddah, Kingdom of Saudi Arabia, using a cluster sampling approach. One-hundred participants were recruited. Participants completed the self-administered Arabic Oral Health Impact Profile (OHIP-14). Five occlusal traits were recorded during clinical examination by three calibrated examiners, including: missing teeth, overjet, crossbite, maximum displacement of contact point, and overbite. Student $t$ test and ANOVA were used to test for statistical significance. There seemed to be a trend for reduced impacts on OHRQoL with age. Gender differences were noticed, but were not statistically significant. Of the five occlusal traits tested, only missing teeth was significantly associated with worse OHRQoL. The study confirms the association between malocclusion and OHQOL in older adults. However, the results need to be examined in other population to confirm the direction and magnitude of this impact.
\end{abstract}

KEYWORDS Oral Health-related Quality of life, malocclusion, adults, Saudi Arabia, Age differences, occlusal traits.

\section{INTRODUCTION}

Oral Health Related Quality of Life (OHRQoL) can be described as the evaluation of one's perception of the oral and oro-facial condition and how this perception affects her/his perception of oral symptoms, functional limitations, emotional wellbeing, and social wellbeing. Increasingly, researchers are adapting OHRQoL measures to assess influence of oro-facial conditions on perceived life quality. In particular, orthodontic

\footnotetext{
* Department of Orthodontics, Faculty of Dentistry, King Abdulaziz University, Jeddah, Saudi Arabia
} 
outcome research lends itself amenable to the notion of OHRQoL, with its strong psychosocial emphasis. Recent systematic reviews summarized studies in this area, and highlighted the need for further research using standardized measures across different populations and various age groups (Andiappan et al., 2015; Dimberg et al., 2015; Liu et al., 2009; Sun et al., 2018a).

The increased demand for orthodontic treatment amongst adults could be attributed to number of reasons, including: the heightened esthetic perception as a result of social media, lengthened lifespan nationally and worldwide, the availability of more aesthetic treatment options such as clear aligner therapy, and possibly, worse impacts of malocclusion on adults' quality of life. That said, current evidence does not support the notion that older adults with malocclusion have worse OHRQoL. As most orthodontic outcome research is mainly focused on examining the relationship between malocclusion/orthodontic treatment need and OHRQoL amongst children, adolescents, and occasionally young adults (Hassan \& Amin, 2010). Very few studies evaluated such a relationship in adults 18 years and above, despite the increased demands for orthodontic treatment among this age group. In fact, a 2009 systematic review identified five studies only assessing the relationship between malocclusion and OHRQoL amongst young adults (Liu et al., 2009). Most studies in this age group focused on the assessment of the impacts of missing teeth or periodontal condition on perceived quality of life.

Several adults instruments have been used to evaluate OHRQoL. The Oral Health Impact Profile (OHIP-49)(Slade \& Spencer, 1994), and its shorter version, the OHIP-14 (Slade, 1997), is one of the earliest and most commonly used OHRQoL measures. It was developed using Locker's conceptual framework (Locker, 1988), and its long version has been translated and validated in Arabic languahe in a sample of Saudi Adults (Al-Jundi et al., 2007), while its short version was validated in a Sudanese sample (Khalifa et al., 2013). The OHIP14 is comprised of 14 items divided on seven subscales. Thus described, the OHIP-14 is a useful tool to assess the impacts of malocclusion, given its good psychometric properties.

Malocclusion is considered a variation of normal, rather than an abnormality. It can be assessed either quantitively or qualitatively. Several indices are used in the literature to assess the severity of malocclusion(Brook \& Shaw, 1989; Lunn et al., 1993; Richmond et al., 1994). Indices usually assign weights or severity scores to relevant occlusal traits. For example, the modified version of the Dental Health Component of the Index of Orthodontic Treatment Need (IOTN.DHC), (Lunn et al., 1993), evaluates particular malocclusion traits including: dental crowding or spacing, crossbite, overjet, overbite, and missing teeth. These five occlusal traits were deemed salient in assessing normative orthodontic treatment needs, and has been assessed in several studies amongst Saudis (Alhummayani \& Taibah, 2018; Hassan, 2006; Taibah \& Al-Hummayani, 2019). However; their association with OHRQoL has not been established in adults.

While the existing body of evidence reports a modest association between malocclusion and OHRQoL amongst children and adolescents, with worse impacts associated with more severe malocclusions, the effects of malocclusion in older adults remain unexplored. This is particularly important given that most orthodontic research on this area is dependent on small convenient samples. Hence, this study aimed to assess how these five occlusal traits affects OHRQoL, as measured by OHIP-14, in a consecutive cluster sample of Saudi adults attending the primary care clinics in the city of Jeddah, Saudi Arabia. Age and gender differences will be also evaluated. 


\section{MATERIALS AND METHODS}

This cross-sectional epidemiological study targeted adults above 18-years-old during their routine visits to primary care dental centers in the city of Jeddah, Kingdom of Saudi Arabia. The local institution's ethical board review approval was obtained, and all patients signed an informed consent after reading the details of the study. A cluster sampling approach was employed. The primary healthcare centers are geographically clustered in five areas of the city (north, south, west, east, and center) by the ministry of health. The inclusion criteria were patients aged 18 years and above, who were medically healthy and were free of any syndromes. Subjects who reported receiving previous orthodontic treatment were excluded. Twenty participants were recruited from each of these areas, resulting in a total sample of one hundred participants. Collected data included demographic information as well as The Arabic OHIP-14, used to assess OHRQoL. Each OHIP question was answered on a five point Likert scale $(0-4)$, and total scores were obtained by summing the scores of each of the 14 questions. Higher OHIP14 scores indicated worse OHRQoL.

Five occlusal traits were recorded during clinical examination by three calibrated examiners, including: missing teeth, orverjet, crossbite, maximum displacement of contact point, and overbite. Clinical examination was completed in the clinic's dental chair, with an examination kit and a millimeter ruler to measure spaces, maximum point displacement, and to record the overjet. The severity of each trait was determined using the cutoff IOTN.DHC scores as a guide. OHIP-14 scores were examined across the severity of five occlusal characteristics. Student $t$ test and ANOVA were used to test for statistical significance.

Each subject was assigned a unique identifier code, and data were coded to obscure any patient's identification information. Data analysis was conducted using the Statistical Package for Social
Sciences program (SPSS Inc., 444 Michigan Avenue, Chicago, USA), version 22. The frequency distribution of each occlusal trait variable and associated mean OHIP scores were calculated. Each malocclusion trait was categorized using IOTN. DHC criteria to give an indication of the severity of the trait. Differences in OHIP-14 scores across the different severity categories of each occlusal traits was assessed using Student $t$ test and ANCOVA. Linear regression analysis was conducted to confirm the association between occlusal traits and OHIP-14 scores after controlling for confounding variables such as age, gender, income, and other occlusal traits. Results were considered statistically significant if the $p$ value was less than $5 \%$.

\section{RESULTS}

A response rate of $100 \%$ was achieved, as all invited patients agreed to participate. Subjects with missing data were excluded during data cleaning. In addition, seven subjects were excluded because they had previous orthodontic treatment, resulting in a total sample of 87 subjects with almost equal male to female distribution (53\% were males), and a mean age of $44.3+/-15.3$ years. The Chronbach's Alpha for the overall OHIP-14 score was 0.88 .

Table 1 reports the OHIP-14 scores of adults for each malocclusion trait. Of the five occlusal traits, statistically significant differences were observed only for missing more than one tooth. The association between OHIP-14 scores and overjet, crossbite, overbite, and displacement of contact point was not statistically significant. Age and gender effect, although not significant were in the expected direction.

The association between missing teeth and OHOP-14 scores remained statistically significant even after controlling for age, gender, income, and other occlusal traits (Table 2). In fact, $12 \%$ of the variance in OHIP-14 scores were explained by missing teeth. 
TABLE (1) Impact of malocclusion traits on OHIP-14 score amongst a sample of Saudi adults

\begin{tabular}{|c|c|c|c|c|c|c|}
\hline \multirow{2}{*}{ Malocclusion criteria } & \multirow{2}{*}{$\begin{array}{c}\text { Malocclusion severity/ } \\
\text { Classification }\end{array}$} & \multirow{2}{*}{ Mean } & \multirow{2}{*}{ SD } & \multicolumn{2}{|c|}{$95 \% \mathrm{CI}$} & \multirow{2}{*}{$p$ value } \\
\hline & & & & $\mathbf{L}$ & $\mathbf{U}$ & \\
\hline \multirow{3}{*}{ Missing Teeth } & No tooth missing & 14.81 & 16.04 & 8.92 & 20.69 & \multirow{3}{*}{$0.034^{*}$} \\
\hline & One tooth missing & 16.64 & 12.67 & 8.12 & 25.15 & \\
\hline & $>1$ tooth missing & 24.25 & 14.67 & 19.29 & 29.21 & \\
\hline \multirow{3}{*}{ Overjet (in mm) } & $0-3.5$ & 19.72 & 14.69 & 14.96 & 24.48 & \multirow{3}{*}{0.950} \\
\hline & $3.6-6$ & 19.00 & 15.65 & 10.95 & 27.05 & \\
\hline & Negative & 17.00 & 21.38 & -36.10 & 70.10 & \\
\hline \multirow{2}{*}{ Crossbite } & Yes & 21.44 & 14.72 & 13.59 & 29.28 & \multirow{2}{*}{0.563} \\
\hline & No & 18.90 & 15.74 & 14.91 & 22.90 & \\
\hline \multirow{4}{*}{$\begin{array}{c}\text { Maximum } \\
\text { displacement of } \\
\text { contact point }\end{array}$} & Displacements $>4 \mathrm{~mm}$ & 19.30 & 12.06 & 10.68 & 27.92 & \multirow{4}{*}{0.299} \\
\hline & Displacements $2.1-4 \mathrm{~mm}$ & 26.00 & 15.56 & 18.50 & 33.50 & \\
\hline & Displacements $1.1-2 \mathrm{~mm}$ & 19.44 & 17.95 & 5.65 & 33.24 & \\
\hline & Displacements less than 1.1 & 17.46 & 14.38 & 11.65 & 23.27 & \\
\hline \multirow{3}{*}{ Overbite (in mm) } & Overbite $>3.5$ & 21.57 & 19.92 & 3.15 & 40.00 & \multirow{3}{*}{0.582} \\
\hline & Overbite $0-3.5$ & 19.81 & 14.81 & 15.51 & 24.11 & \\
\hline & Negative $<0$ & 28.00 & 11.52 & 9.67 & 46.33 & \\
\hline \multirow{3}{*}{ Age (in years) } & $19-40$ & 22.43 & 14.78 & 16.70 & 28.16 & \multirow{3}{*}{0.142} \\
\hline & $41-60$ & 17.88 & 15.58 & 12.45 & 23.32 & \\
\hline & $>60$ & 12.09 & 12.87 & 3.44 & 20.74 & \\
\hline \multirow{2}{*}{ Gender } & Male & 17.68 & 15.24 & 12.87 & 22.49 & \multirow{2}{*}{0.299} \\
\hline & Female & 21.35 & 15.71 & 16.11 & 26.59 & \\
\hline
\end{tabular}

*Indicates a statistically significant difference as confirmed by ANOVA

TABLE (2) ANCOVA model evaluating the association between OHIP-14 scores, after controlling for age, gender, income, and overjet

\begin{tabular}{|l|c|c|c|c|c|}
\hline \multicolumn{5}{|c|}{ Tests of Between-Subjects Effects } \\
\hline \multicolumn{3}{|c|}{ Dependent Variable: Total score } \\
\hline Source & Type III Sum of Squares & df & Mean Square & F & Sig. \\
\hline Corrected Model & $3324.362 \mathrm{a}$ & 9 & 369.374 & 1.897 & .075 \\
\hline Intercept & 2940.776 & 1 & 2940.776 & 15.101 & .000 \\
\hline gender & 53.004 & 1 & 53.004 & .272 & .604 \\
\hline age & 726.928 & 1 & 726.928 & 3.733 & .059 \\
\hline Income & 138.379 & 1 & 138.379 & .711 & .403 \\
\hline Missing teeth & 2436.903 & 2 & 1218.452 & 6.257 & .004 \\
\hline Overjet & 358.268 & 2 & 179.134 & .920 & .405 \\
\hline Missing teeth*Overjet & 392.560 & 2 & 196.280 & 1.008 & .373 \\
\hline Error & 9347.655 & 48 & 194.743 & & \\
\hline Total & 35197.000 & 58 & & & \\
\hline Corrected Total & 12672.017 & 57 & & & \\
\hline
\end{tabular}

a. $R$ Squared $=.262($ Adjusted $R$ Squared $=.124)$ 


\section{DISCUSSION}

This manuscript reports on the influence of five occlusal traits on OHRQOL in a sample of adult patients attending the primary care clinics in the city of Jeddah Saudi Arabia. Of the five traits tested, only missing teeth had significant impacts on quality of life in this population. Other occlusal traits such as crossbite, overbite, and displacement of contact point did not seem to have an impact on OHRQoL. The direction and magnitude of OHRQoL impacts in relation to missing teeth, overbite, and crossbite were in the expected direction. For example, subjects with crossbite had worse OHRQoL compared to those without a crossbite. Similarly, subjects with deep overbite or openbite had worse OHRQoL compared to those with normal overbite. The lack of statistical significance might be due to sampling variations across the different categories. That said, changes in overjet and displacement of contact points were not consistent. This suggested that these malocclusion traits may have different impacts on OHRQoL. The insignificant associations between increased overjet and OHRQoL is not supported by other studies, which denotes the significant effect increased overjet has on quality of life of 10-20-year-olds (Obilade et al., 2016), and severe crowding and severe protrusion resulting in poorer OHRQoL (Jung, 2015).

Research in younger age groups suggest that differences between subjective and objective orthodontic treatment need can be explained by understanding how malocclusion impacts OHRQoL. Previous research demonstrates a clear inverse association of malocclusion with OHRQOL (Andiappan et al., 2015; Dimberg et al., 2015; Liu et al., 2009; Sun et al., 2018a). However, the correlation coefficients of this association varied as factors such as age and culture changed. Moreover, several researchers indicated that this relationship could be ameliorated by other variables such as self-esteem and psychological wellbeing (Agou et al., 2008, 2011; Benson et al., 2015; Claudino \& Traebert, 2013; Clijmans et al., 2015; Dos Santos et al., 2017; Jung, 2015), and was considered relatively low (Andiappan et al., 2015).

The results from this study shed light on possible differences in the older age group (above 18-yearold), compared to young adults. In a previous study assessing the relationship between malocclusion and OHRQoL in Saudi young adults (Hassan \& Amin, 2010), it was found that patients with normative orthodontic treatment need suffered from several impacts on quality of life. This calls for further investigations to fully understand how OHRQoL is perceived amongst older adults. For example, though not statistically significant, there seemed to be a general tendency to report less impacts as subjects grew older. This could be attributed to adopting psychological coping mechanisms, being able to afford treatment, or becoming less concerned with dentofacial appearance and more occupied with other health issues. Masood et al., reported that younger people report higher levels of impact (Masood et al., 2013). Gender differences were also not significant. That said, females reported slightly worse OHRQoL, which corroborates the findings from previous studies (Masood et al., 2013; Rusanen et al., 2010). In addition, a cross-sectional study of 18-year-olds in Hong Kong, found that household income and malocclusion impacted OHRQoL the worst, with malocclusion primarily affecting the psychological discomfort and psychological disability domains. Supporting the findings of our study, others found no relationship between gender, periodontal status and caries and young adults' OHRQoL (Sun et al., 2018b).

The association between missing teeth and overall OHIP-14 scores, remained significant $(p<0.05)$ even after controlling for confounders in the regression model (Table 2). This is not surprising, in this age group. Previous studies of older adults highlight the devastating effect of having unrestored missing teeth on quality of life (Anbarserri et al., 2020). In our study, about $12 \%$ of the variance of OHIP-14 scores was explained by missing teeth. This association warrants further investigation to evaluate 
the effect of teeth number and teeth position on OHRQoL.

The OHIP-14 demonstrated excellent psychometric properties in this study, with a Chronbach's Alpha for the overall OHIP-14 score of 0.88 , indicating excellent internal consistency. A previous meta-analysis of OHIP-14 scores documented its ability to discriminate between subjects with or without malocclusion/orthodontic treatment need (Andiappan et al., 2015).

Inherent to the cross-sectional study design employed, there are some limitations. The directions and magnitude of the association cannot be confirmed. Also, data were collected from one city in Saudi Arabia, and the sample size is relatively small. Hence, the generalizability of the results is limited. It might have been better to employ a case-control design to evaluate the impact of each occlusal trait on OHRQoL. That said, it is very difficult to find cases with isolated malocclusion features. It was, therefore, legitimate to assess each factor while controlling for the effect of other occlusal traits using regression analysis. The next step would be to use a validated index to assess the severity of malocclusion and its relationship with OHRQoL.

Using OHRQoL instruments to complement normative measures in evaluating the impact of occlusal traits on OHRQoL need to be made (Kok et al., 2004). This study highlights the high impacts of having missing teeth on the quality of life of older adults. Ministry of Health policy makers need to consider these findings in resource allocation and planning.

\section{CONCLUSION}

This epidemiological study evaluated the effect of five occlusal traits on OHRQoL among adults in Saudi Arabia. Of all the factors evaluated, missing teeth seem to have the largest impact on quality of life.

\section{ACKNOWLEDGMENT}

I would like to acknowledge Dr. Talal Zahid for his help in planning and conducting the study, and for facilitating the communication with the Ministry of Health and the primary care centers. Also, I would like to thank Dr. Fawaz Pullishery for his statistical assistance.

\section{Disclosure}

No potential conflict of interest was reported by the author.

\section{REFERENCES}

1. Agou, S., Locker, D., Muirhead, V., Tompson, B., \& Streiner, D. L. (2011). Does psychological well-being influence oral-health-related quality of life reports in children receiving orthodontic treatment? American Journal of Orthodontics and Dentofacial Orthopedics: Official Publication of the American Association of Orthodontists, Its Constituent Societies, and the American Board of Orthodontics, 139(3), 369-377. https://doi.org/10.1016/j. ajodo.2009.05.034

2. Agou, S., Locker, D., Streiner, D. L., \& Tompson, B. (2008). Impact of self-esteem on the oral-health-related quality of life of children with malocclusion. American Journal of Orthodontics and Dentofacial Orthopedics : Official Publication of the American Association of Orthodontists, Its Constituent Societies, and the American Board of Orthodontics, 134(4), 484-489. https://doi. org/10.1016/j.ajodo.2006.11.021

3. Alhummayani, F. M., \& Taibah, S. M. (2018). Orthodontic treatment needs in Saudi young adults and manpower requirements. Saudi Medical Journal, 39(8), 822-828. https://doi.org/10.15537/smj.2018.8.22337

4. Al-Jundi, M. A., Szentpétery, A., \& John, M. T. (2007). An Arabic version of the Oral Health Impact Profile: Translation and psychometric properties. International Dental Journal, 57(2), 84-92. https://doi.org/10.1111/j.1875$595 x .2007 . t b 00443 . x$

5. Anbarserri, N. M., Ismail, K. M., Anbarserri, H., Alanazi, D., AlSaffan, A. D., Baseer, M. A., \& Shaheen, R. (2020). Impact of severity of tooth loss on oral-health-related quality of life among dental patients. Journal of Family 
Medicine and Primary Care, 9(1), 187-191. https://doi. org/10.4103/jfmpc.jfmpc_909_19

6. Andiappan, M., Gao, W., Bernabé, E., Kandala, N.-B., \& Donaldson, A. N. (2015). Malocclusion, orthodontic treatment, and the Oral Health Impact Profile (OHIP-14): Systematic review and meta-analysis. The Angle Orthodontist, 85(3), 493-500. https://doi.org/10.2319/051414-348.1

7. Benson, P. E., Da'as, T., Johal, A., Mandall, N. A., Williams, A. C., Baker, S. R., \& Marshman, Z. (2015). Relationships between dental appearance, self-esteem, socio-economic status, and oral health-related quality of life in UK schoolchildren: A 3-year cohort study. European Journal of Orthodontics, 37(5), 481-490. https://doi. org/10.1093/ejo/cju076

8. Brook, P. H., \& Shaw, W. C. (1989). The development of an index of orthodontic treatment priority. European Journal of Orthodontics, 11(3), 309-320. https://doi.org/10.1093/ oxfordjournals.ejo.a035999

9. Claudino, D., \& Traebert, J. (2013). Malocclusion, dental aesthetic self-perception and quality of life in a 18 to 21 year-old population: A cross section study. BMC Oral Health, 13, 3. https://doi.org/10.1186/1472-6831-13-3

10. Clijmans, M., Lemiere, J., Fieuws, S., \& Willems, G. (2015). Impact of self-esteem and personality traits on the association between orthodontic treatment need and oral health-related quality of life in adults seeking orthodontic treatment. European Journal of Orthodontics, 37(6), 643-650. https://doi.org/10.1093/ejo/cju092

11. Dimberg, L., Arnrup, K., \& Bondemark, L. (2015). The impact of malocclusion on the quality of life among children and adolescents: A systematic review of quantitative studies. European Journal of Orthodontics, 37(3), 238247. https://doi.org/10.1093/ejo/cju046

12. Dos Santos, P. R., Meneghim, M. de C., Ambrosano, G. M. B., Filho, M. V., \& Vedovello, S. A. S. (2017). Influence of quality of life, self-perception, and self-esteem on orthodontic treatment need. American Journal of Orthodontics and Dentofacial Orthopedics : Official Publication of the American Association of Orthodontists, Its Constituent Societies, and the American Board of Orthodontics, 151(1), 143-147. https://doi.org/10.1016/j.ajodo.2016.06.028

13. Hassan, A. H. (2006). Orthodontic treatment needs in the western region of Saudi Arabia: A research report. Head \& Face Medicine, 2, 2. https://doi.org/10.1186/1746160X-2-2
14. Hassan, A. H., \& Amin, H. E.-S. (2010). Association of orthodontic treatment needs and oral health-related quality of life in young adults. American Journal of Orthodontics and Dentofacial Orthopedics : Official Publication of the American Association of Orthodontists, Its Constituent Societies, and the American Board of Orthodontics, 137(1), 42-47. https://doi.org/10.1016/j.ajodo.2008.02.024

15. Jung, M.-H. (2015). An evaluation of self-esteem and quality of life in orthodontic patients: Effects of crowding and protrusion. The Angle Orthodontist, 85(5), 812-819. https://doi.org/10.2319/091814.1

16. Khalifa, N., Allen, P. F., Abu-bakr, N. H., \& Abdel-Rahman, M. E. (2013). Psychometric properties and performance of the Oral Health Impact Profile (OHIP-14s-ar) among Sudanese adults. Journal of Oral Science, 55(2), 123-132. https://doi.org/10.2334/josnusd.55.123

17. Kok, Y. V., Mageson, P., Harradine, N. W. T., \& Sprod, A. J. (2004). Comparing a quality of life measure and the Aesthetic Component of the Index of Orthodontic Treatment Need (IOTN) in assessing orthodontic treatment need and concern. Journal of Orthodontics, 31(4), 312-318; discussion 300-301. https://doi. org/10.1179/146531204225020625

18. Liu, Z., McGrath, C., \& Hägg, U. (2009). The impact of malocclusion/orthodontic treatment need on the quality of life. A systematic review. The Angle Orthodontist, 79(3), 585-591. https://doi.org/10.2319/042108-224.1

19. Locker, D. (1988). Measuring oral health: A conceptual framework. Community Dental Health, 5(1), 3-18.

20. Lunn, H., Richmond, S., \& Mitropoulos, C. (1993). The use of the index of orthodontic treatment need (IOTN) as a public health tool: A pilot study. Community Dental Health, 10(2), 111-121.

21. Masood, Y., Masood, M., Zainul, N. N. B., Araby, N. B. A. A., Hussain, S. F., \& Newton, T. (2013). Impact of malocclusion on oral health related quality of life in young people. Health and Quality of Life Outcomes, 11, 25. https:// doi.org/10.1186/1477-7525-11-25

22. Obilade, O. A., Sanu, O. O., \& Costa, O. O. da. (2016). Impact of three malocclusion traits on the quality of life of orthodontic patients. International Orthodontics, 14(3), 366-385. https://doi.org/10.1016/j.ortho.2016.07.003

23. Richmond, S., Roberts, C. T., \& Andrews, M. (1994). Use of the Index of Orthodontic Treatment Need (IOTN) in assessing the need for orthodontic treatment pre- and 
post-appliance therapy. British Journal of Orthodontics, 21(2), 175-184. https://doi.org/10.1179/bjo.21.2.175

24. Slade, G. D., \& Spencer, A. J. (1994). Development and evaluation of the Oral Health Impact Profile. Community Dental Health, 11(1), 3-11.

25. Sun, L., Wong, H. M., \& McGrath, C. P. J. (2018a). Association Between the Severity of Malocclusion, Assessed by Occlusal Indices, and Oral Health Related Quality of Life: A Systematic Review and Meta-Analysis. Oral Health \& Preventive Dentistry, 16(3), 211-223. https://doi. org/10.3290/j.ohpd.a40761

26. Sun, L., Wong, H. M., \& McGrath, C. P. J. (2018b). The factors that influence oral health-related quality of life in young adults. Health and Quality of Life Outcomes, 16(1), 187. https://doi.org/10.1186/s12955-018-1015-7

27. Taibah, S. M., \& Al-Hummayani, F. M. (2019). Agreement and association between normative and subjective orthodontic treatment need using the Index of Orthodontic Treatment Need. Journal of Orthodontic Science, $8,1$. https://doi.org/10.4103/jos.JOS_87_18 